\section{Cuidado de pessoas vivendo com HIV na atenção primária à saúde: reconfigurações na rede de atenção à saúde?}

\author{
Care for persons living with HIV in primary health \\ care: reconfigurations of the healthcare network?
}

\section{Cuidado de personas viviendo con VIH en la atención primária de salud: ¿reconfiguraciones en la red de atención a la salud?}

Eduardo Alves Melo 1,2

Rafael Agostini 3,4

Jorginete de Jesus Damião 5

Sandra Lúcia Filgueiras 6

Ivia Maksud 3 doi: 10.1590/0102-311X00344120

\section{Resumo}

Este estudo analisa a recente experiência de descentralização do cuidado às pessoas vivendo com HIV para a atenção primária à saúde (APS) do Rio de Janeiro, Brasil. Para tanto, é subsidiado por uma pesquisa qualitativa em duas unidades de APS de uma região da cidade do Rio de Janeiro, a partir de observação participante, grupos focais com profissionais de saúde e entrevistas individuais com estes e com usuários, realizados entre os anos de 2018 e 2019. Os dados revelam, ainda que de modo heterogêneo, a implementação avançada de testes rápidos e ampliação do número de pessoas acompanhadas, os problemas e inovações nos modos de acesso a APS, os aspectos na gestão do sigilo e centralidade do médico de família e comunidade no cuidado. Problematizamos, a partir disso, paradoxos relacionados à noção de território operada na APS (ampliando, ao mesmo tempo, acesso e vulnerabilidades ligadas ao risco de estigma) bem como tensões entre as necessidades dos usuários e os modos de organização do serviço. Concluímos que o cuidado das pessoas vivendo com HIV pode ser, ao mesmo tempo, um analisador da APS (interrogando suas práticas e bases) e um dispositivo de mudança (mediante rearranjos organizacionais e de práticas de cuidado), porém, atentando para a impossibilidade de garantir atenção integral apenas através da APS, e aos desafios de ordem não apenas técnica, mas também política, organizativa, ética e moral que se colocam neste processo.

Atenção Primária à Saúde; HIV; Assistência Integral à Saúde

\author{
Correspondência \\ E. A. Melo \\ Departamento de Administração e Planejamento em Saúde, \\ Escola Nacional de Saúde Pública Sergio Arouca, \\ Fundação Oswaldo Cruz. \\ Rua Leopoldo Bulhões 1480, 7o andar, Rio de Janeiro, RJ \\ 21041-210, Brasil. \\ eduardo.melo@ensp.fiocruz.br

\footnotetext{
1 Escola Nacional de Saúde Pública Sergio Arouca, Fundação Oswaldo Cruz, Rio de Janeiro, Brasil.

2 Instituto de Saúde Coletiva, Universidade Federal Fluminense, Niterói, Brasil.

3 Instituto Nacional de Saúde da Mulher, da Criança e do Adolescente Fernandes Figueira, Fundação Oswaldo Cruz, Rio de Janeiro, Brasil.

4 Faculdade de Medicina, Universidade Cidade de São Paulo, São Paulo, Brasil.

5 Instituto de Nutrição, Universidade do Estado do Rio de Janeiro, Rio de Janeiro, Brasil.

6 Gerência Estadual de IST/Aids, Secretaria Estadual de Saúde do Rio de Janeiro, Rio de Janeiro, Brasil.
} 


\section{Introdução}

No desenho assistencial do SUS, a atenção primária à saúde (APS) deve estar distribuída amplamente no território e aparece como base das redes de atenção à saúde (RAS), formadas também por outras modalidades de ações e serviços organizados em regiões de saúde 1.

A APS tem sido formulada internacionalmente a partir de alguns atributos ditos essenciais e outros derivados, na expectativa de que ela seja o primeiro contato do usuário com o sistema de saúde e que coordene o seu trânsito assistencial por toda a RAS ao longo do tempo e em diversas condições de saúde 2. A assistência prestada pela APS deve, ainda, considerar não apenas a dimensão individual dos usuários, mas também aquelas referentes à família e à comunidade 2.

Desde o final dos anos de 1970, com a Conferência de Alma-Ata, a APS vem ganhando mais atenção no plano global. No caso brasileiro, esse movimento se destacou em meados dos anos de 1990, quando se instituiu o Programa Saúde da Família (PSF). No entanto, na virada dos anos 2000, este espaço de atenção ganha centralidade ainda maior na agenda pública brasileira, até que, em 2006, passa a figurar oficialmente no texto da Política Nacional de Atenção Básica (PNAB) como prioritária na reorganização do modelo técnico-assistencial através da Estratégia Saúde da Família (ESF) 3.

A PNAB, que disciplina a organização da APS no Brasil, prevê que as unidades básicas de saúde (UBS) devem organizar suas práticas de atenção individual e coletiva a partir de uma base territorial definida, com adscrição de clientela. Outra diretriz é a conformação de equipes multiprofissionais, que incluem - além de médicos, enfermeiros e técnicos de enfermagem - a figura de um membro da comunidade que tem como uma das suas funções o elo entre a instituição e os sujeitos: os agentes comunitários de saúde (ACS). Esta atribuição foi mantida na última versão da PNAB, em 2017, apesar dos seus retrocessos 4 .

Especificamente no caso do HIV, a atuação da APS é historicamente tímida, pois no Brasil a assistência às pessoas vivendo com HIV se consolidou como responsabilidade dos serviços especializados, com a testagem vinculada aos Centros de Testagem e Aconselhamento e a prevenção realizada em parceria com organizações não governamentais (ONG). Gradativamente, todavia, a APS foi aumentando sua participação nessa seara, embora limitada à prevenção e, a partir de 2012, à testagem 5.

Na segunda década dos anos 2000, diversas inovações no manejo da infecção pelo HIV foram incorporadas no SUS como a terapia combinada, a recomendação de tratamento antirretroviral a todas as pessoas vivendo com HIV e a profilaxia pré-exposição. Como pano de fundo destas mudanças está a tentativa de materializar localmente a estratégia global 90/90/90, que prevê metas para o fim dos níveis epidêmicos da aids até 20306 .

Nesse cenário, o Ministério da Saúde passou a recomendar modelos descentralizados, em que, através de uma estratificação de riscos, a assistência às pessoas vivendo com HIV, incluindo aquelas em uso de antirretroviral, fosse realizada também na APS, diante de sua capilaridade territorial e papel destacado no acesso 7,8 .

A cidade do Rio de Janeiro, Brasil, foi uma das primeiras a iniciar este movimento, ainda em 2013, a partir de um protocolo provisório adotado pela gestão municipal 9. Embora o acompanhamento de pessoas vivendo com HIV na APS seja apenas uma possibilidade e não uma obrigação, em 2019, aproximadamente 50\% das pessoas vivendo com HIV em acompanhamento neste município o faziam na APS 10. Sendo verdade que a descentralização apresenta potencialidades, como a ampliação do acesso, também traz consigo desafios importantes, como a gestão do cuidado e do sigilo em particular 11.

Neste sentido, o objetivo deste artigo é analisar a recente experiência de descentralização do cuidado de pessoas vivendo com HIV para a APS no Rio de Janeiro, partindo da hipótese de que tal processo pode funcionar como um dispositivo analisador 12 de algumas bases epistemológicas e organizativas da APS. 


\section{Metodologia}

A investigação que subsidia este artigo foi produzida no âmbito da pesquisa intitulada $O$ Cuidado às Pessoas com HIV/AIDS na Rede de Atenção à Saúde, no período de abril de 2018 a outubro de 2019, no contexto da rede municipal de saúde do Rio de Janeiro. Trata-se de um estudo de método etnográfico 13 que envolveu trabalho de campo contendo várias técnicas como: observação participante em UBS e em atividades desenvolvidas no território, entrevistas semiestruturadas em profundidade, com usuários e diversas categorias profissionais, e grupos focais com ACS.

Os participantes foram selecionados processualmente, com o desenrolar do trabalho de campo, sendo a técnica snowball recorrentemente utilizada. Para a produção desse artigo, além dos dados provenientes de observações e de conversas informais, nos beneficiamos mais diretamente da análise de 30 entrevistas e de dois grupos focais (com cerca de 10 participantes cada), que tiveram em média duração de uma e duas horas, respectivamente, foram gravados em áudio e posteriormente transcritos. Nos orientamos pelo critério de saturação de dados para a finalização do campo, a despeito das dificuldades para atingir tal feito 14 .

Os dados foram produzidos no contexto de duas unidades de APS indicadas pela gestão municipal, nas quais havia, à época, 13 das quase 75 equipes de saúde da família (EqSF) de uma região central da cidade com aproximadamente 300 mil habitantes. A abordagem aos participantes foi feita em seus espaços de trabalho, por seis pesquisadores que se alternavam no campo, munidos de roteiros, contendo como tópico principal a descentralização do cuidado às pessoas vivendo com HIV. Diários e notas de campo eram produzidos e discutidos em reuniões mensais da equipe de pesquisa, assim como aspectos biográficos, idiossincráticos e marcadores sociais que possam ter moldado os encontros, mas que não serão alvo de reflexão neste texto, por motivos de espaço.

O processo de análise dos dados se deu em diálogo com referenciais teóricos sobre HIV e aids na APS, cuidado e integralidade 11,15,16,17,18,19,20,21,22, interrogando elementos da APS a partir da observação sobre o que o cuidado às pessoas vivendo com HIV evidenciava ou provocava nela. O conjunto de dados produzidos foi analisado considerando as seguintes dimensões: acesso à testagem na APS, escolha do serviço para acompanhamento e fluxos de pessoas vivendo com HIV na APS e gestão do cuidado das pessoas vivendo com HIV na APS. Estas dimensões foram elaboradas a partir da análise do conteúdo dos dados da pesquisa e dos referenciais adotados, tendo a problemática e o propósito do estudo como guias.

A pesquisa foi aprovada pelos Comitês de Ética em Pesquisa da Escola Nacional de Saúde Pública Sergio Arouca, Fundação Oswaldo Cruz e da Secretaria Municipal de Saúde do Rio de Janeiro (SMS-RJ) recebendo, respectivamente, a aprovação de números 2.309 .404 e 2.559 .884 . A participação dos interlocutores observou todos os aspectos éticos previstos nas normativas brasileiras sobre consentimento, sigilo e confidencialidade das informações obtidas em pesquisa com seres humanos.

\section{Resultados e discussão}

Antes de adentrar nos resultados, caracterizaremos brevemente os contextos de sua produção. Tratase de duas unidades com portes e perfis diferentes, sendo a primeira, UBS 1, formada por oito EqSF que cobriam cerca de 25 mil pessoas e a outra, UBS 2, de perfil misto, conjugando três EqSF com a atenção básica dita tradicional sendo responsável por aproximadamente 14 mil pessoas. Ambas funcionavam como campos de estágio para estudantes de graduação e a primeira também contava com Residência em Medicina de Família e Comunidade.

A despeito da localização central, tais unidades conviviam com a violência armada presente nos territórios, habitados majoritariamente por população de baixa renda, notadamente nos morros, em que vive a maior parte da população cadastrada. Quanto às pessoas vivendo com HIV com diagnóstico conhecido, havia aproximadamente 100 delas no território da primeira unidade e 84 no da segunda. A UBS 2 já dispensava antirretrovirais e realizava exames de monitoramento do CD4 e carga viral, ao passo que a UBS 1 incorporou tais exames no curso da pesquisa e seguiu acessando antirretrovirais em uma unidade próxima. No que tange aos encaminhamentos, feitos através de sistema informa- 
tizado de regulação, ambas tinham facilidade quando se tratava de infectologistas, podendo haver dificuldade para outros exames e especialidades.

\section{Acesso à testagem na APS}

O acesso tem sido considerado, há algum tempo, como um importante aspecto dos sistemas de saúde, contemplando não apenas dimensões espaciais, mas também culturais e organizacionais, com implicações sobre a qualidade dos serviços e a aceitação dos usuários 23,24. Especificamente na APS, a temática tem sido permeada pelas ações programáticas, pelo acolhimento à demanda espontânea e, mais recentemente, pelo acesso avançado 25 . A necessidade de ampliação do acesso das pessoas vivendo com HIV ao diagnóstico e ao tratamento, por sua vez, tem sido evocada pelos tomadores de decisão como razão para pensar novos arranjos e ofertas de cuidado no campo do HIV e aids 6 .

Nas duas unidades de APS da nossa pesquisa, destacou-se o grau de incorporação de testes rápidos na rotina, tanto para o HIV quanto para outras infecções sexualmente transmissíveis (IST) como a sífilis e as hepatites B e C (guardando aqui a excepcionalidade da transmissão por via sexual desta última). Como podemos ver abaixo, há relatos que dão conta de certa facilidade na realização destes exames quando demandados pelos usuários. A segunda fala evidencia a possibilidade, inclusive, de que os usuários possam realizar a testagem em qualquer uma das unidades de APS do município, desde que manifestem o desejo de realizar tal procedimento para as ACS durante o acolhimento, pois, em geral, usuários "fora de área" são encaminhados para a unidade de referência do seu território de moradia.

"Assim, se chegou ao acolhimento e quer fazer o teste rápido, aí, a gente coloca na planilha, e esse teste rápido já faz diretamente com a enfermeira (...) e tem as médicas que também pedem internamente devido algum relato (...)” (ACS da UBS 2).

"Pode até fazer fora de área [também] (...), [é] a mesma coisa, vai fazer um cadastro rápido lá [no acolhimento] (...), passa por uma consulta e vai fazer [o teste]. $E$ [aí] você pode escolher [e dizer]: olha, eu não moro na área, mas se caso der positivo eu quero fazer o tratamento aqui, eu estou me sentido bem, fui bem atendido, fui bem acolhido (...) é um direito dele" (ACS da UBS 1).

A pesquisa observou ainda que, com o passar do tempo, a testagem foi absorvida na rotina de ofertas das unidades, se espraiando para além do público, em nome do qual se ensejou nacionalmente a sua descentralização: as gestantes 26 . Apesar de tal alargamento, estas mulheres continuam sendo parcela expressiva dos usuários que acessam a testagem, como revela a fala de uma das ACS da UBS 1 durante o grupo focal: "na minha equipe, a maior parte dos casos que aparecem estão relacionados à gravidez".

Neste sentido, constatamos também diferentes formas de testagem, desde aquelas ligadas à iniciativa dos usuários até ações territoriais esporádicas, protagonizadas pelas EqSF. Estas últimas se realizam com cuidados adicionais de maneira que o sigilo possa ser garantido, de modo geral, e com relação aos atores do tráfico de drogas, de modo específico. Aliás, diversos profissionais defendem a importância de que essas ações - bem como aquelas de prevenção - sejam ampliadas, mas indicam obstáculos para tanto, como os tabus que atravessam os temas da sexualidade, a estigmatização do HIV e a violência.

Apesar de já incorporada à rotina das unidades, pudemos observar que a prática de testagem na APS coexiste com preocupações relativas à possibilidade de quebra de sigilo sobre a realização ou sobre o resultado do teste, em função dos formatos de organização da testagem nas unidades incluindo espaços físicos, horários e profissionais envolvidos - facilitando a identificação de pessoas residentes no bairro que estão se testando, bem como a observação de suas reações após receberem a comunicação do resultado.

Houve relatos indicando que os testes rápidos já foram vistos pelos profissionais como um "trabalho a mais”, além de terem surgido dúvidas sobre a sua acurácia para a confirmação. Reportou-se dificuldade de aconselhamento quando da comunicação de um resultado positivo para o HIV. Um dos usuários entrevistados também relata dificuldade com o aconselhamento: após exame solicitado por uma interna, a médica o teria comunicado o diagnóstico de forma agressiva e brusca enfatizando a possibilidade dele "ir para a cadeia" caso saísse "dando para todo mundo sem camisinha", o que poderia também agravar o seu quadro clínico. A literatura aponta o comprometimento da efetividade do aconselhamento quando há baixa dialogicidade desta prática nos serviços, com abordagens normativas da conduta dos usuários, nas quais a sexualidade aparece apenas como risco 27,28,29. 
A utilização de testes rápidos parece ter feito com que o diagnóstico de HIV passasse a estar mais presente (e em tempo real) nas unidades, colocando o tema na agenda das equipes com maior destaque e, possivelmente, fazendo com que soubessem de mais pessoas vivendo com HIV nos seus territórios de atuação. Tendemos a acreditar que este processo contribuiu, ainda que parcialmente, para o acompanhamento de pessoas vivendo com HIV na APS. Ao mesmo tempo, percebe-se a importância de aprimorar não apenas o aconselhamento (que se dá em geral nos consultórios), mas também o próprio modo de organização da oferta de testagem na APS.

\section{Escolha do serviço para acompanhamento e fluxos de pessoas vivendo com HIV na APS}

Ao considerar a assistência às pessoas vivendo com HIV que já conhecem o seu diagnóstico pudemos observar a possibilidade de serem acompanhadas na APS ou num serviço especializado, conforme sua preferência. O primeiro tipo de fluxo tende a acontecer em função do vínculo já existente entre usuário e equipe de APS ou da abordagem inicial dos profissionais (especialmente o médico) a partir do diagnóstico, mesmo em casos cuja indicação de acompanhamento com infectologista é clara. Um dos médicos da UBS 1, por exemplo, sustentou o vínculo construído anteriormente para fazer o acompanhamento de um usuário com infecção por tuberculose e HIV. Nas palavras dele: "a gente segurou na mão na hora que estava chorando, a gente vai mandar pro especialista esse cara?".

Usuários que escolhem a APS parecem fazê-lo, como percebemos entre interlocutores da UBS 1, em função da relação com os profissionais: "dá uma atenção, me passa até uma segurança. É ele que está sendo meu porto seguro"; do modelo de cuidado "...[o médico] vai se deslocar para a comunidade, correr um risco e tal. Isso não é pra qualquer um (...), me senti escolhido depois desse dia”; da abordagem mais ampliada de saúde "as palavras dele [do médico de família e comunidade] me confortam" e "eu consigo conversar com ele meus problemas, meu dia a dia..."; do vínculo "é o meu médico particular, aqui é minha clínica"; e da confiança "devido à eficiência dele, o Pedro Ernesto até me dispensou [do acompanhamento da tuberculose]". A satisfação com o primeiro contato em cenários de integração da APS na assistência às pessoas vivendo com HIV foi encontrada em experiências de outros países 30 .

O segundo tipo de fluxo, por outro lado, tende a se dar quando a condição clínica de maior risco configura indicação para encaminhamento a um serviço especializado, a exemplo de gestantes com HIV; ou quando o usuário prefere ser acompanhado num local com menos risco de seu diagnóstico ser conhecido por familiares ou vizinhos, o que em alguns casos pode gerar, inclusive, violências e mesmo expulsão do território, como nos reportaram alguns interlocutores. Também identificamos a ocorrência deste tipo de fluxo em situações de dúvida, por parte dos usuários, sobre a capacidade da APS, por não ser um lugar "especializado". Um dos usuários entrevistados na UBS 1, que fazia tratamento na rede de saúde suplementar até perder o plano de saúde e procurar a UBS, nos relatou se sentir mais amparado em ser atendido por infectologista por este ser especialista, com "estudo", "focado naquela área".

No decorrer da pesquisa a SMS-RJ publicou nota técnica permitindo também às pessoas vivendo com HIV a escolha de qualquer unidade de APS para seu acompanhamento independente do seu local de moradia. Se até então a alternativa era ser acompanhado na APS no território de moradia ou no serviço especializado, com a nova diretriz, qualquer unidade de APS do município é elegível e aquela que é escolhida passa a ser a sua unidade de referência, exceto para ações de atenção domiciliar 31.

Esta medida gerou divergências entre os profissionais, com alguns indicando que este arranjo dificulta o acompanhamento. Apesar das vozes dissonantes, e ainda que não tenhamos observado muitas situações deste tipo, esta flexibilização da lógica territorial nos pareceu uma inovação significativa por parte da gestão. De modo corrente na realidade brasileira - diferente do que ocorre em algumas experiências internacionais - não é comum a escolha da UBS e/ou da equipe de referência na APS ser feita pelo usuário, sendo em geral definida a partir do seu endereço de moradia e, eventualmente, do local de trabalho; confunde-se, por vezes, adscrição (medida de ordem administrativa) com vinculação (marcada pela construção de relação de afetividade e confiança) 32 .

A possibilidade da escolha sobre onde prefere ser acompanhado nos remete às reflexões de Cecílio 33. O autor salienta que a configuração tecnológica estabelecida nos serviços de saúde deve ser sempre definida a partir da necessidade de cada sujeito naquele determinado momento e que "a 'hierarquia' de 
importância do consumo das tecnologias não a estabelecemos unicamente nós, técnicos, mas, também, as pessoas com suas necessidades reais" 33 (p. 115).

Para Cecílio 33, portanto, as demandas e necessidades dos usuários deveriam presidir a organização dos serviços. Nesta mesma perspectiva alinham-se as contribuições de Mattos 34 sobre as noções de qualidade das práticas de saúde, que devem ter "como eixo, a garantia do acesso adequado e oportuno às ações e serviços de saúde que tenham potência de responder às necessidades das pessoas” 34 (p. 778), reforçando a importância da efetivação do princípio da integralidade na conquista desta qualidade almejada.

Há que se ressaltar ainda, que não apenas o vínculo do usuário com os profissionais parece ser importante para o acompanhamento e adesão ao tratamento, mas também as implicações territoriais, que podem resultar em facilitação de acesso, mas também em mais (sensação de) insegurança para as pessoas vivendo com HIV, pelo risco de discriminação e estigma ao morar e ser acompanhado na mesma localidade, onde familiares, conhecidos e outros atores também transitam e se cuidam. Isto reforça a necessidade de compreender o território - uma categoria-chave para a saúde coletiva e para a APS - para além do espaço físico, considerando sua dinâmica social de produção e uso, sua dimensão existencial e mesmo a sua multiplicidade por vezes paradoxal 15,35.

Uma vez sendo acompanhada numa UBS, há outros momentos de acesso também importantes para uma pessoas vivendo com HIV. Percebemos, nas duas unidades, usuários com HIV passando por fluxos em geral idênticos àqueles dos demais usuários. Na configuração organizacional das UBS do Rio de Janeiro, os usuários, ao adentrarem o serviço, se dirigem ao que se chamam de "baias", organizadas por equipe, e com presença de ACS encarregados de receber os usuários, respondendo inicialmente a suas demandas e direcionando seus fluxos. Em uma das unidades operava-se com um misto de agendamentos prévios dos usuários e de demanda espontânea. Na outra, predominava o acesso por demanda espontânea, através do chamado "acesso avançado", que vem sendo experimentado em outros países e em algumas cidades brasileiras 25.

Especialmente na busca por demanda espontânea, usuários precisam informar nas baias o motivo da ida à unidade, o que podia ser embaraçoso para as pessoas vivendo com HIV, dado que, no geral, o ACS é o profissional sobre o qual mais incidem receios quanto ao sigilo diagnóstico. Além disso, comumente há muitos outros usuários-moradores na sala de espera que podem ter curiosidade, ouvir conversas que acontecem nas baias, ou ainda querer saber o motivo de alguns "conhecidos" estarem na unidade. Pode-se ver, com isso, que para pessoas vivendo com HIV com o legítimo e fundado receio da estigmatização e dos seus efeitos na vida, o espaço da sala de espera e este formato de fluxo para acesso pode ser vivenciado com certo sofrimento, a ponto mesmo de comprometer, no limite, a aceitação daquele serviço e o acompanhamento 16.

Ouvimos críticas de alguns profissionais à dificuldade de acompanhamento de pessoas vivendo com HIV gerada pelo acesso avançado (sem agendamento de consultas subsequentes), assim como ouvimos também estratégias desenvolvidas por eles diretamente junto a elas para evitar desconfortos na sua chegada à unidade, como, por exemplo, fornecer o número de telefone e pedir que entrem em contato por WhatsApp antes de irem à unidade, ou que avisem nas baias simplesmente que o médico pediu seu retorno à unidade (sem necessidade de detalhar motivos). Neste sentido, podemos falar de uma espécie de aliança entre alguns médicos de família e comunidade e pessoas vivendo com HIV, por vezes operada com "suspensão parcial" de determinadas regras, podendo facilitar o acesso e favorecer a construção de vínculos.

Apesar deste movimento, a observação processual de aparente "fluxo privilegiado" e/ou de outras situações e registros (como papéis com receitas, encaminhamentos e resultados de exames) nos pareceram ir gerando "suspeitas", de modo indiciário, pelos ACS, fazendo com que muitos destes acabem sabendo do diagnóstico dos usuários da sua área, mesmo sem estes e sem os demais profissionais da equipe o terem revelado. O mesmo pode acontecer a partir da organização da coleta de CD4 e carga viral (por vezes em dias e horários específicos) bem como na fila para retirada de medicação antirretroviral na farmácia da UBS.

Destacamos ainda que o mencionado "fluxo privilegiado", para além de não garantir completamente o segredo sobre o diagnóstico, revela a existência de equívocos importantes entre o que sejam ações que buscam gerar equidade e aquelas que de fato favorecem privilégios e desigualdade de acesso. Diante desta questão, nos remetemos a Kalichman \& Ayres 21, para quem a integralidade - vista como combinação de aspectos técnicos e organizativos com elementos de sentido político em termos de 
direitos e cidadania - faz-se fundamental para que a universalidade e a equidade da atenção à saúde se realizem de modo pleno 21 .

Nesse sentido, defende-se que a construção de um cuidado integral impõe a execução de ações estratégicas que viabilizem, àqueles que vivenciam situações de maior vulnerabilidade, a igualdade no acesso e na qualidade dos serviços de atenção à saúde. Ayres 20 (p. 11) enfatiza que "o princípio da universalidade nos impulsiona a construir o acesso para todos, o da equidade nos exige pactuar com todos o que cada um necessita, mas a integralidade nos desafia a saber e fazer o 'quề' e 'como' pode ser realizado em saúde para responder universalmente às necessidades de cada um...”.

Estas problematizações sobre o acesso das pessoas vivendo com HIV na APS reforçam a necessidade de compreender o tema na sua complexidade, para além da proximidade física do usuário com um serviço, bem como suas especificidades na APS para se operar de fato um acesso facilitado e favorável aos usuários. Como estamos tratando da APS num sistema universal, não plenamente efetivado e sob constrangimentos estruturais, a discussão sobre flexibilidade no acesso, anteriormente realizada, não significa a inexistência de regras e fluxos formais nem mudanças no acesso a cada caso, mas a atenção sensível aos casos em que as normas podem colidir com as necessidades dos usuários e a reflexão sobre arranjos alternativos, visando garantir o acesso com qualidade.

\section{Gestão do cuidado das pessoas vivendo com HIV na APS}

Embora o acesso seja, em si, ainda um grande desafio presente no SUS (sobretudo à atenção especializada), o modo como se cuida, após ultrapassar algumas barreiras de acesso na entrada do serviço de saúde, também é algo fundamental, não só pelas complexas relações entre demanda e oferta, como também porque o campo do cuidado é atravessado por distintas forças, dimensões e interesses, inclusive aqueles que operam na micropolítica dos encontros entre trabalhadores e usuários 18 . Neste sentido, nossa pesquisa pôde captar alguns elementos que nos parecem relevantes, ainda que estivéssemos mais atentos à organização e gestão do cuidado do que às práticas de cuidado em si.

Considerando o trabalho multiprofissional que tanto marca a APS brasileira, chamou-nos atenção a grande centralidade da figura do médico no cuidado às pessoas vivendo com HIV na experiência estudada, em que pese a relevância observada dos profissionais de enfermagem na prevenção, testagem e aconselhamento, e dos ACS no apoio à gestão do cuidado de alguns casos e, indiretamente, em situações como a entrega de fichas de agendamento de exames ou consultas especializadas. Isto é diferente, por exemplo, do que acontece no acompanhamento de crianças pequenas, de gestantes, de usuários com tuberculose ou com hipertensão e diabetes, em que o cuidado direto é compartilhado entre médicos e enfermeiros.

Tal diferença parece se dever ao fato de o acompanhamento de pessoas vivendo com HIV na APS ser relativamente recente no município; outro motivo parece ser o fato de que alguns desses usuários por vezes procuram a UBS apenas para renovação da receita e, em algumas delas, não querem sequer passar por consulta. É possível que a necessidade do sigilo e da legitimação deste processo de acompanhamento também tenham reforçado o cuidado médico, dando mais segurança e evitando que alegações ou desconfianças sobre qualidade técnica sejam mais um elemento a colocar em dúvida a capacidade da APS. Cabe assinalar, ainda, a importância verificada dos protocolos clínicos como ferramentas frequentemente utilizadas pelos médicos no cuidado às pessoas vivendo com HIV, orientando o manejo, bem como os critérios de encaminhamento.

Desta forma, esta centralidade do médico nos faz indagar os alcances e limites do cuidado às pessoas vivendo com HIV na APS em termos de integralidade, seja pelo fato de que o trabalho em equipe multiprofissional pode ampliar a capacidade de compreensão e análise sobre problemas, demandas e necessidades de saúde, seja pelas implicações diretas que o acompanhamento a partir do médico (como agente central) tem sobre os tipos de oferta de cuidado agenciadas, por mais ampliada que seja a clínica do médico de família e comunidade.

De fato, percebemos fragilidades, por exemplo, nas práticas de vigilância (notadamente monitoramento e avaliação), a ponto de as equipes, como regra, não saberem o número de pessoas vivendo com HIV por elas acompanhadas, embora isto também possa derivar de limites do sistema de informação disponibilizado pela gestão, mas que, por outro, é diferente do que acontece com outros públicos e condições já consagrados na APS, como as gestantes. 
Além disso, ainda que a sensibilidade e compromisso dos médicos de família e comunidade tenham se mostrado de grande relevância para o acesso e o sigilo das pessoas vivendo com HIV, e que haja um movimento de qualificação técnica para o manejo clínico, não visualizamos iniciativas abordando a questão da diversidade e das práticas sexuais, nem ações visando combater o estigma e a discriminação, ou fortalecer a autoestima e sociabilidade das pessoas vivendo com HIV, que são também aspectos relevantes do cuidado às pessoas vivendo com HIV, inclusive segundo normativas oficiais 36. Isto não se restringe ao fato de o trabalho ser uni ou multiprofissional, mas também à necessidade de intervenções nos planos grupal e coletivo, e que extrapolem os limites do bairro (onde um grupo de convivência formado por pessoas vivendo com HIV daquele território torna-se mais difícil, por exemplo).

Na unidade com Residência em Medicina da Família e Comunidade e presença forte de especialistas nessa área, observamos também um duplo movimento destes profissionais. Por um lado, entendem a responsabilização pela assistência às pessoas vivendo com HIV como "uma naturalidade da especialidade" - referindo-se à Medicina de Família e Comunidade - já que "quando você abraça aquela especialidade, abraça com tudo que ela vem". Nesta direção, sustentam que não é razoável "triar uma pessoa por patologia” uma vez que a APS “acompanha a pessoa”, independentemente da condição sorológica e, portanto, "o fato de simplesmente ter HIV não é justificativa para [o usuário] acompanhar na atenção secundária”. Apontam ainda que muitas vezes já têm vínculo com o usuário e que isto poderia ser perdido encaminhando-os ao infectologista.

Além disso, esses profissionais parecem ver também no cuidado às pessoas vivendo com HIV uma oportunidade de marcar a diferença do médico de família e comunidade em relação a outras especialidades - chamadas por eles de "focais" - e de ampliar seu próprio reconhecimento. Este movimento pode ser compreendido se considerarmos que a Medicina de Família e Comunidade ainda se encontra em processo de divulgação e legitimação no Brasil 37, por vezes apoiado em argumentos ou formulações que buscam caracterizá-la como outra medicina, estruturada a partir de uma epistemologia híbrida e integral 38 .

\section{Considerações finais}

Partindo da intenção de analisar a experiência de descentralização do cuidado de pessoas vivendo com HIV na APS, este estudo identificou, nas unidades de APS do Rio de Janeiro, onde foi realizado: implementação avançada de testes rápidos acompanhada de dificuldades no aconselhamento e na revelação do diagnóstico; ampliação do número de pessoas vivendo com HIV acompanhadas na APS, ainda que de modo heterogêneo; problemas e inovações nos modos de acesso à APS para as pessoas vivendo com HIV e na gestão do sigilo (particularmente em relação aos ACS e aos moradores do bairro); centralidade do médico de família e comunidade no cuidado às pessoas vivendo com HIV.

Neste cenário, destacam-se paradoxos relacionados à noção de território operada na APS (ampliando, ao mesmo tempo, acesso e vulnerabilidades ligadas ao risco de estigma) bem como tensões entre as necessidades de pessoas vivendo com HIV e os modos de organização da APS. Como pudemos perceber, o cuidado de pessoas vivendo com HIV pode ser, ao mesmo tempo, um analisador da APS (interrogando algumas das suas práticas e bases) e um dispositivo de mudança (mediante rearranjos organizacionais e de práticas de cuidado), porém, atentando para a impossibilidade de garantir atenção integral apenas através da APS, e aos desafios de ordem não apenas técnica, mas também política, organizativa, ética e moral que se colocam neste processo.

A nosso ver, a adoção deste tipo de experiência, notadamente na realidade heterogênea e desigual do Brasil, requer prudência (considerando-a como possibilidade e não como obrigatoriedade), ainda que apresente elementos novos e promissores em termos de acesso e vinculação na APS, inspirando novas experimentações e estudos, inclusive para pessoas que não vivem com HIV.

É oportuno assinalar que não abordamos, neste artigo, resultados relativos à interação entre médicos de família e comunidade e infectologistas, tema importante que será objeto de artigo dedicado à interface entre APS e atenção especializada no cuidado às pessoas vivendo com HIV no cenário de descentralização dos cuidados, em razão da especificidade e complexidade desta dimensão. 


\section{Colaboradores}

Todos os autores contribuíram na concepção e delineamento do estudo, coleta e análise dos dados, redação e revisão final do manuscrito.

\section{Informações adicionais}

ORCID: Eduardo Alves Melo (0000-0001-58814849); Rafael Agostini (0000-0001-8071-9362); Jorginete de Jesus Damião (0000-0001-6591-3474); Sandra Lúcia Filgueiras (0000-0003-2971-734X); Ivia Maksud (0000-0002-3465-151X).

\section{Referências}

1. Magalhães Jr. HM. Redes de Atenção à Saúde: rumo à integralidade. Divulg Saúde Debate 2014; 52:15-37.

2. Starfield B. Atenção primária: equilíbrio entre necessidades de saúde, serviços e tecnologia. Brasília: Organização das Nações Unidas para a Educação, a Ciência e a Cultura/Ministério da Saúde; 2002.

3. Giovanella L, Mendonça MHM. Atenção primária em saúde. In: Giovanella L, Escorel S, Lobato LVC, Noronha JC, Carvalho AI, organizadores Políticas e sistema de saúde no Brasil. Rio de Janeiro: Editora Fiocruz; 2008. p. 575-626.

4. Melo EA, Mendonça MHM, Oliveira JR, Andrade GCL. Mudanças na Política Nacional de Atenção Básica: entre retrocessos e desafios. Saúde Debate 2018; 42(spe1):38-51.

5. Zambenedetti G, Silva RAN. O paradoxo do território e os processos de estigmatização no acesso ao diagnóstico de HIV na atenção básica em saúde. Estud Psicol (Natal) 2015; 20:229-40.

6. Granich R, Williams B, Montaner J, Zuniga JM. 90-90-90 and ending AIDS: necessary and feasible. Lancet 2017; 390:341-3.

7. Departamento de DST, Aids e Hepatites Virais, Secretaria de Vigilância em Saúde, Ministério da Saúde. Caderno de boas práticas em HIV/ AIDS na atenção básica. Brasília: Ministério da Saúde; 2014

8. Departamento de DST, Aids e Hepatites Virais, Secretaria de Vigilância em Saúde, Ministério da Saúde. 5 passos para a implementação do manejo da infecção pelo HIV na atenção básica. Brasília: Ministério da Saúde; 2014.

9. Programa das Doenças Infecciosas Sexualmente Transmissíveis, Secretaria Municipal de Saúde do Rio de Janeiro. Protocolo para introdução do atendimento ao HIV/Aids na Rede Primária de Saúde do Município do Rio de Janeiro. Rio de Janeiro: Secretaria Municipal de Saúde do Rio de Janeiro; 2013.

10. Silva G. Situação da linha de cuidado à pessoa vivendo com HIV/AIDS na cidade do Rio de Janeiro. In: Maksud I, Melo EA, Rocha F, Damião JJ, organizadores. Anais do Seminário Coro de Vozes numa teia de significados: sobre o cuidado às pessoas vivendo com HIV/ AIDS na Rede de Atenção à Saúde. Rio de Janeiro: Instituto Nacional de Saúde da Mulher, da Criança e do Adolescente Fernandes Figueira; Escola Nacional de Saúde Pública Sergio Arouca, Fundação Oswaldo Cruz/Instituto de Saúde Coletiva, Universidade Federal Fluminense/Fundação de Amparo à Pesquisa do Estado do Rio de Janeiro/Fundação casa de Rui Barbosa; 2020. p. 13-9.

11. Melo EA, Maksud I, Agostini R. Cuidado, HIV/ Aids e atenção primária no Brasil: desafio para a atenção no Sistema Único de Saúde? Rev Panam Salud Pública 2018; 42:e151.

12. Rossi A, Passos E. Análise Institucional: revisão conceitual e nuances da pesquisa-intervenção no Brasil. Revista EPOS 2014; 5:156-81. 
13. Victora CG, Knauth DR, Hassen MNA. Pesquisa qualitativa em Saúde: uma introdução ao tema. Porto Alegre: Tomo Editorial; 2000.

14. Minayo MCS. Amostragem e saturação em pesquisa qualitativa: consensos e controvérsias. Revista Pesquisa Qualitativa 2017; 5:1-12.

15. Zambenedetti G. O paradoxo do território e os processos de estigmatização da AIDS na atenção básica em saúde [Tese de Doutorado]. Porto Alegre: Universidade Federal do Rio Grande do Sul; 2014.

16. Zambenedetti G, Silva RAN. O paradoxo do território e os processos de estigmatização no acesso ao diagnóstico de HIV na atenção básica em saúde. Estud Psicol (Natal) 2015; 20:229-40.

17. Zambenedetti G, Silva RAN. Descentralização da atenção em HIV-Aids para a atenção básica: tensões e potencialidades. Physis (Rio J.) 2016; 26:785-806

18. Cecílio LCO. A morte de Ivan Ilitch, de Leon Tolstói: elementos para se pensar as múltiplas dimensões da gestão do cuidado. Interface (Botucatu) 2009; 13 Suppl 1:545-55.

19. Mattos RA. Os sentidos da integralidade: algumas reflexões acerca dos valores que merecem ser defendidos. In: Pinheiro R, Mattos RA, organizadores. Os sentidos da integralidade na atenção e no cuidado à saúde. Rio de Janeiro: Instituto de Medicina Social, Universidade do Estado do Rio de Janeiro; 2001. p. 39-64.

20. Ayres JRCM. Organização das ações de atenção à saúde: modelos e práticas. Saúde Soc 2009; 18 Suppl 2:11-23.

21. Kalichman AO, Ayres JRCM. Integralidade e tecnologias de atenção à saúde: uma narrativa sobre contribuições conceituais à construção do princípio da integralidade no SUS. Cad Saúde Pública 2016; 32:e00183415.

22. Merhy EE, Feuerwerker LCM, Santos MLM, Bertussi DC, Baduy RS. Rede básica, campo de forças e micropolítica: implicações para a gestão e cuidado em saúde. Saúde Debate 2019; 43:70-83.

23. Assis MMA, Jesus WLA. Acesso aos serviços de saúde: abordagens, conceitos, políticas e modelo de análise. Ciênc Saúde Colet 2012; 17:2865-75

24. Sanchez RM, Ciconelli RM. Conceitos de acesso à saúde. Rev Panam Salud Pública 2012; 31:260-8.

25. Rocha SA, Bocchi SCM, Godoy MF. Acesso aos cuidados primários de saúde: revisão integrativa. Physis (Rio J.) 2016; 26:87-111.

26. Ministério da Saúde. Portaria no 1.459 , de 24 de junho de 2011. Institui, no âmbito do Sistema Único de Saúde - SUS - a Rede Cegonha. Diário Oficial da União 2011; 27 jun.

27. Araújo CLF, Camargo Jr. KR. Aconselhamento em DST/HIV: repensando conceitos e prática. Rio de Janeiro: Folha Carioca; 2004.

28. Mora C, Monteiro S, Moreira COF. Formação, práticas e trajetórias de aconselhadores de centros de testagem anti-HIV do Rio de Janeiro, Brasil. Interface (Botucatu) 2015; 19:1145-56.
29. Zakabi D, d'Oliveira AFPL, Teixeira RR. Encontros e conversas durante a testagem anti-HIV na atenção primária em São Paulo. Essentia 2018; 19:63-75.

30. Odeny TA, Penner J, Lewis-Kulzer J, Leslie $\mathrm{HH}$, Shade SB, Adero W, et al. Integration of HIV Care with primary health care services: effect on patient satisfaction and stigma in rural Kenya. AIDS Res Treat 2013; 2013:485715.

31. Subsecretaria de promoção, Atenção Primária e Vigilância em Saúde, Secretaria Municipal de Saúde do Rio de Janeiro. Ofício Circular S/ SUBPAV/SAP no 09/2019. Acesso aos serviços de atenção Primária à Saúde (APS) por pessoas vivendo com HIV/AIDS (PVHA). Rio de Janeiro: Secretaria Municipal de Saúde do Rio de Janeiro; 2019. (Nota Técnica 04).

32. Gutiérrez AC, Guerrero AVP, Cunha GT. Reflexões sobre a atenção básica e a Estratégia de Saúde da Família. In: Campos GWS, Guerrero AVP, organizadores. Manual de práticas de atenção básica. Saúde ampliada e compartilhada. São Paulo: Hucitec Editora; 2008. p. $132-254$.

33. Cecílio LCO. As necessidades de saúde como conceito estruturante. In: Pinheiro R, Mattos RA, organizadores. Os sentidos da integralidade na atenção e no cuidado à saúde. Rio de Janeiro: Instituto de Medicina Social, Universidade do Estado do Rio de Janeiro; 2001. p. 113-26.

34. Mattos RA. Princípios do Sistema Único de Saúde (SUS) e a humanização das práticas de saúde. Interface (Botucatu) 2009; 13 Suppl 1:771-80.

35. Gondim GMM, Monken M. O uso do território na APS. O uso do território na atenção primária à saúde. In: Mendonça MM, Matta GC, Gondim R, Giovanella L, organizadores. Atenção primária à saúde no Brasil: conceitos, práticas e pesquisa. Rio de Janeiro: Editora Fiocruz; 2018. p. 143-75.

36. Departamento de Doenças de Condições Crônicas e Infecções Sexualmente Transmissíveis, Secretaria de Vigilância em Saúde, Ministério da Saúde. Protocolo clínico e diretrizes terapêuticas para atenção integral às pessoas com infecções sexualmente transmissíveis (IST). Brasília: Ministério da Saúde; 2020.

37. Coelho Neto GC, Antunes VH, Oliveira A. A prática da Medicina de Família e Comunidade no Brasil: contexto e perspectivas. Cad Saúde Pública 2019; 35:e00170917.

38. Bonet O. Os médicos da pessoa: um olhar antropológico sobre a medicina de família no Brasil e na Argentina. Rio de Janeiro: 7Letras; 2014. 


\section{Abstract}

The study analyzes the recent experience with decentralization of care for persons living with HIV in primary health care (PHC) in Rio de Janeiro, Brazil. The study is backed by a qualitative survey in PHC units in a region of the city of Rio de Janeiro, based on participant observation, focus groups with healthcare workers, and individual interviews with the latter and with patients in 2018 and 2019. The data show (although with uneven distribution) the advanced implementation of rapid tests and expansion of the number of persons followed by the services, besides problems and innovations in the forms of access to PHC, aspects in the management of confidentiality, and the central role of family and community physicians in the care. Based on the above, we problematize paradoxes related to the notion of territory operated in PHC (meanwhile expanding access and vulnerabilities associated with the risk of stigma) as well as tensions between patients' needs and modes of the services' organization. We conclude that care for persons living with HIV can serve both to analyze PHC (studying its practices and foundations) and as a device for change (through organizational rearrangements and patient care practices), but without ignoring the possibility of guaranteeing comprehensive care solely through PHC or the challenges in this process, not only technical, but also political, organizational, ethical, and moral.

Primary Health Care; HIV; Comprehensive Health Care

\section{Resumen}

Este estudio analiza la reciente experiencia de descentralización del cuidado a las personas viviendo con VIH en la atención primaria de salud (APS), en Río de Janeiro, Brasil. Para ello, es apoyado por una investigación cualitativa en dos unidades de APS de una región de la ciudad de Río de Janeiro, a partir de observación participante, grupos focales con profesionales de salud y entrevistas individuales con ellos y con usuarios, realizados entre los años de 2018 y 2019. Los datos revelan, incluso que, de modo heterogéneo, la implementación avanzada de test rápidos y la ampliación del número de personas acompañadas, los problemas e innovaciones en los modos de acceso a APS, los aspectos en la gestión de la confidencialidad y centralidad del médico de familia y comunidad en el cuidado. Problematizamos, a partir de esto, paradojas relacionadas con la noción de territorio operada en la APS (ampliando, al mismo tiempo, acceso y vulnerabilidades vinculadas al riesgo de estigma), así como tensiones entre las necesidades de los usuarios y los modos de organización del servicio. Concluimos que el cuidado de las personas viviendo con VIH puede ser, al mismo tiempo, un analizador de la APS (interrogando sus prácticas y bases) y un dispositivo de cambio (mediante reordenamiento organizacionales y de prácticas de cuidado), no obstante, considerando la imposibilidad de garantizar la atención integral solamente a través de la APS, y los desafíos de orden no exclusivamente técnico, sino también político, organizativo, ético y moral que se sitúan en este proceso.

Atención Primaria de Salud; VIH; Atención Integral à Salud
Recebido em 10/Dez/2020

Versão final reapresentada em 30/Mar/2021

Aprovado em 29/Abr/2021 